\title{
VALOR DIAGNOSTICO DEL EXAMEN DEL FONDO DE OJO EN PACIENTES EMBARAZADAS CON HIPERTENSION ARTERIAL
}

\author{
Dr. Fernando Sánchez T.* \\ Dr. Jesús A. Gómez P.* \\ Dr. Hugo Guevara J.* \\ Dr. Armando Santamaría $H$.*
}

Uno de los hechos evidentes en la fisiopatología de la Toxemia Gravídica Aguda (TGA) es el vasoespasmo arteriolar generalizado, el cual explica el cortejo sintomático de la enfermedad: hipertensión arterial, edemas, proteinuria, convulsiones. Además, la vasoconstricción, con la consiguiente isquemia, es también causa preponderante de sus principales complicaciones.

En la práctica el diagnóstico de la TGA procura hacerse utilizando en conjunto los procedimientos ordinarios de examen. Uno de estos es, precisamente, la visualización de los vasos retinianos. A pesar de que Finnerty $(5,6)$ describiera como característico de la TGA un aspecto brillante, húmedo, de toda la retina, más valor se ha dado a las alteraciones del calibre vascular $(1,9,12$, $14,17)$. Por tratarse de un proceso hipertensivo agudo los cambios que con frecuencia se observan son los espasmos generalizados o focalizados de las arteriolas, que parecen sean debidos a modificaciones funcionales; también es posible encontrar hemorragias "en llama" y exudados algodonosos.
Es cosa aceptada, además, que el examen del fondo de ojo es un buen recurso para identificar aquellos procesos que, a diferencia de la TGA, han evolucionado por tiempo más o menos largo y que producen alteraciones orgánicas de los vasos $(1,3$, $8,9,12)$.

Por otra parte, está plenamente demostrado que la TGA ocasiona cambios característicos en los glomérulos renales de las mujeres que la padecen y que su severidad está de acuerdo con la gravedad clínica de la enfermedad $(2,4,13,15)$. De ahí que el estudio histopatológico del tejido renal obtenido por biopsia sea el único procedimiento que permite diagnosticar, de manera inobjetable, la ocurrencia y gravedad de la TGA.

Como las investigaciones que se han llevado a cabo sobre los cambios vasculares retinianos en el curso de la TGA se han basado solo en el diagnóstico clínico de la enfermedad -que no es siempre fácil-, es de suponer que esos resultados no refle-

* Del Departamento de Obstetricia y Ginecología y del Departamento de Patología. Facultad de Medicina de la Universidad Nacional. 
jen la realidad. Unicamente Pollak y cols. (14), en su importante monografía sobre "Riñón en la Toxemia Gravídica", relacionaron los hallazgos oftalmoscópicos con los cambios renales y pudieron deducir que el espasmo de las arteriolas retinianas es el hecho más significativo.

La presente comunicación tiene por objeto dar a conocer nuestras observaciones respecto al valor del fondo de ojo en pacientes cuyo embarazo estuvo complicado con hipertensión arterial de etiología aclarada por el estudio histopatológico de tejido renal obtenido por biopsia.

\section{Material y Métodos}

Este trabajo, hecho en el Servicio de Toxemias del Instituto Materno Infantil de Bogotá, comprende el estudio de 107 mujeres que padecieron hipertensión arterial en el curso de su embarazo. Pese a que cada enferma fue estudiada detenidamente, tanto en el aspecto clínico como de laboratorio, solo tuvimos en cuenta el diagnóstico histopatológico.

El examen de fondo de ojo, exploración de rutina del Servicio, fue practicado al ingreso de la enferma o algunos pocos días después. Las personas que lo hicieron fueron siempre las mismas, familiarizadas con el procedimiento e identiifcadas con los criterios establecidos para valorar los cambios observados. Estos fueron clasificados en los siguientes grupos:

Grado O: Normal.

Grado I: Vasoconstricción generalizada o localizada.

Grado IA: Presencia de arteriolas en hilos de cobre o plata.

Grado 11: Alteraciones en los cruces arteriolovenulares, es decir, compresión u obliteración de la vena.
Grado III' Hemorragias "en Ilama" - "en estrías", cercanas a la papilla, solas o asociadas a exudados.

Grado IV: Papiledema bilateral.

Por su parte, la biopsia renal fue ejecutada con la aguja de Vin-Silverman-Franklin, en posición prono. El tejido fue fijado en formalina al 10\% e incluído en parafina; en cortes de 3 micras de espesor se hicieron coloraciones de hematoxilina-eosina, PAS y tricrómica de Mallory. La evaluación histopatológica se hizo encasillando los cambios, tanto toxémicos como hipertensivos, en uno de los grupos propuestos por Pollak (14), usando el microscopio de luz. Por considerar que el grupo con alteraciones mínimas descrito por él se presta a una interpretación muy subjetiva, no fue tenido en cuenta. Para la toxemia el diagnóstico se basó en los cambios observados en los glomérulos: edema de la pared capilar, "espesamiento" de la membrana basal, isquemia e hipercelularidad. Para la hipertensión en modificaciones de los vasos: hiperplasia de la íntima, hipertrofia de la media, necrosis, perielastosis, etc. Los cambios toxémicos o hipertensivos se clasificaron en cuatro grupos, así:

Grado O: Normal.

Grado 1: Cambios ligeros.

Grado II: Cambios moderados.

Grado III: Cambios severos.

\section{Resultados}

Como señalamos atrás, el diagnóstico de la enfermedad de fondo se basó exclusivamente en las modificaciones histopatológicas del riñón. Por este procedimiento se encontró que 58 pacientes tenían únicamente TGA; en otras 5 había además pielonefritis 
crónica; en 26 la TGA se asociaba a cambios vasculares de hipertensión crónica, y en 7, a más de estos, a focos de pielonefritis. Finalmente, en 3 se trataba de glomérulonefritis crónica.

Para relacionar los cambios renales con lo observado en el fondo de ojo, veremos por aparte cada uno de estos grupos.

1. Toxemia Gravídica Aguda TGA: De 63 pacientes con TGA, en 55 $(87,3 \%)$ el fondo de ojo fue completamente normal (Grado O), a pesar de que en 8 de ellas los cambios de toxemia eran muy intensos (Grado III) y de que en 5 había además lesiones de pielonefritis crónica.

En $5(8 \%)$ había espasmo arteriolar (Grado I), siendo en 3 los cambios toxémicos de Grado I y en 2 de Grado II.

En 3 casos $(4,7 \%)$ se apreciaron cambios representativos de lesión orgánica (Grado la), uno de ellos asociado a exudados algo donosos (Grados $|A-||i|)$.

2. Enfermedad vascular hipertensiva crónica y TGA asociada: De las 33 mujeres así clasificadas, en 27 $(81,8 \%)$, incluyendo 7 con pielonefritis, la oftalmoscopia puso de presente lesiones vasculares orgánicas (Grado II), con exudados o hemorragias en 5 de ellas (Grados II-III). En las 6 restantes, 4 tenían fondo de ojo normal y en 2 había espasmos arteriolares (Grado I).

3. Enfermedad vascular hipertensiva’ crónica:

De estos 8 casos en 7 había lesión orgánica de los vasos retinianos (Grados IA y II). En otro con cambios oculares II-III y IV, la biopsia reveló necrosis arteriolar fibrinoide, caracte- rística de la fase maligna de la hipertensión.

4. Glomérulonefritis crónica:

En los 3 casos de enfermedad renal crónica por glomérulonefritis había lesiones vasculares de tipo orgánico (Grado III), en uno de ellos asociadas a hemorragias (Grados II-III).

\section{Comentarios y Conclusiones}

Como su nombre lo indica, la TGA cs un proceso de aparición súbita durante el curso del embarazo y que compromete todo el árbol arteriolar, por acción de sustancias presoras no identificadas todavía $(7,16)$. Uno de los sitios donde el clínico puede observar este hecho es en la superficie retiniana - 0 en el lecho vascular de la conjuntiva- (10), siendo posible advertir constricciones generalizadas - localizadas, calificadas como de tipo funcional. Ya desde 1933 Wagener (17) había establecido que la variabilidad de los estrechamientos vasculares en la TGA y su desaparición rápida después del parto, inducían a tener que aceptar que eran manifestaciones funcionales más bien que lesiones angioescleróticas.

Si es cierto que la hipertensión arterial de cualquier origen, mantenida durante un tiempo largo provoca en las paredes arteriolares cambios orgánicos de tipo esclerótico (8), la aparición de arteriolas en hilos de plata o de cobre, la obliteración de las venas en el sitio que las cruzan las arterias, o las hemorragias retinianas, serán representación definida de dicho proceso. Por eso es que si se observan en una paciente embarazada con sindrome hipertensivo arterial, ha de pensarse que la hipertensión existía desde mucho antes de iniciarse el embarazo $(9,12,17)$. 
Basándonos, pues, en diagnósticos exclusivamente histopatológicos - por estudio del tejido renal- y relacionándolos con las modificaciones arteriolares —valoradas por medio del oftalmoscopio_, podemos manifestar que el $87 \%$ de los casos de TGA pura las arteriolas retinianas no exhiben cambios suficientes como para ser observados al examen clínico, pese a que la enfermedad se haya catalogado histopatológicamente como muy severa. Esto está en desacuerdo con los hallazgos de Agüero y Torres (1), quienes en el $72 \%$ de los casos de preeclampsia observaron cambios cuya severidad estuvo en relación directa con el cuadro clínico.

En cambio, en el $86 \%$ de las enfermas con lesiones vasculorrenales de hipertensión, solas o asociadas a toxemia o a enfermedad renal primaria, se apreciaron claramente modificaciones arteriolares orgánicas en el fondo de ojo.

De lo anterior podemos concluir que en los casos de TGA es lo común que no se encuentren cambios vasculares retinianos, y que si existen sean del tipo funcional. Cualquier alteración calificada como orgánica permite sospechar, casi con certeza, que hay una enfermedad hipertensiva crónica. Además, la presencia de hemorragias con o sin exudados (fondo de ojo Grado III) es más frecuente en la hipertensión crónica de cualquier etiología que en la TGA. Nosotros las observamos en un caso de TGA (1/63) y en 6 de hipertensión crónica $(6 / 44)$.

\section{Resumen}

A 107 mujeres embarazadas que padecían hipertensión arterial les fue practicada biopsia renal percutánea y los hallazgos histopatológicos fueron relacionados con los cambios vas- culares encontrados al examen del fondo de ojo.

En el 87,3 por 100 de las pacientes con Toxemia Gravídica Aguda ( $T$ GA) el fondo de ojo fue normal, a pesar de que los cambios renales de toxemia fueron muy severos.

En el 86 por 100 de las embarazadas con Enfermedad Hipertensiva Crónica (EHC) se apreciaron alteraciones orgánicas de los vasos retinianos.

Las hemorragias, con o sin exudados, se observaron con una frecuencia mucho mayor en los casos de EHC que en aquellos con TGA.

\section{BIBLIOGRAFIA}

1 AGUERO, O. y TORRES C. "Fondo de ojo en las toxemias del embarazo". Progr. Obst. y Gin. 2: 257, 1959.

2 ALTCHEK, A.: "Electron microscopy of renal biopsies in toxemia of pregnancy". J.A.M.A. 175: 791, 1961.

3 BAHAMON, J. "Fondo de ojo en toxicosis gravídica". Tribuna Médica. 3: 1, 1964.

4 DIECKMANN, W. J.; POTTER, E. L. Y MCCARTNEY, CH. P. "Renal biopsies from patients with toxemia of pregnancy". Am. J. Obst. \& Gynec. 73: 1, 1957.

5 FINNERTY, F. A.: "Toxemia del embarazo". Cl. Med. N. A. Marzo. 487, 1961.

6 FINNERTY, F. A. Jr. "Toxemia of pregnancy as seen by an internist: an analysis of 1081 patients". Ann. Int. Med. 44: 358, 1956.

7 HUNTER, CH. A. y HOWARD, W. F. "A pressor substance (hysterotonin) ocurring in toxemia". Am. J. Obst. \& Gynec. 79: $838,1960$.

8 KIRKENDALL, W. M. "Examen del fondo de ojo en pacientes con hipertensión arterial". Publicacin de Merck Sharp \& Dohme, 1967.

9 LANDESMAN, R.; DOUGLAS, G. y SNYDER, S. S. "Retinal changes in the toxemias of pregnancy". Am. J. Obst. \& Gynec. 63 : 16, 1952.

10 LANDESMAN, R.; GORDON, R. Y HOLZE, E. "The bulbar conjunctival vascular bed in the toxemias of pregnancy". Am. J. Obst. \& Gynec. 68: 170, 1954. 
11 McGAUGHEY, L. H. "The cause of the blood and vascular alterationes of normal pregnancy and preeclampsia - eclampsia". Am. J. Obst. \& Gynec. 64: 1268, 1952.

12 MUSSEY, R. D. Y MUNDELL, B. J. "A guide in the management of the toxic hypertensive syndrome of pregnancy". Am. J. Obst. \& Gynec. 37: 30, 1939.

14 POLLAK, V. E. y NETTLES, J. B. "Kydney in toxemia of pregnancy". Medicin 39: 469, 1960.
15 SPARGO, B.; MCCARTNEY, CH. P. Y WINEMILLER, R. "Glomerualr capillary endotheliosis in toxemia of pregnancy". Arch. of Path. 68: 593.

16 TATUM, H. J. Y MULE, J. G. "The hypertensive action of blood from patients with preeclampsia". Am. J. Obst. \& Gynec. 83: 1028, 1962.

17 WAGENER, H. P. "Arterioles of the retina in toxemia of pregnancy". JAMA. 101: $1.380,1960$. 